Thus by (2.6) and (2.7)

$$
t_{n}^{(2)} \sim-(d / \pi) \log n
$$

Hence by (2.3), (2.5), and (2.8), we have

$$
t_{n} \sim(d / \pi) \log n,
$$

which completes the proof. ${ }^{7}$

Ravenshaw College

'A note dealing with the analogous result for the differentiated Fourier series proved under the condition corresponding to (1.5) has been accepted by this journal. This condition may be replaced by the one corresponding to (1.4).

\title{
ON SUFFICIENT CONDITIONS FOR OVERCONVERGENCE ${ }^{1}$
}

\section{T. J. RIVLIN}

Ostrowski has shown that a Taylor series $f(z)=\sum_{n=0}^{\infty} a_{n} z^{n}$ with finite nonzero radius of convergence overconverges in a neighborhood of each point of the circle of convergence at which $f(z)$ is regular if $a_{n}=0$ for $m_{k}<n<n_{k}, m_{k}, n_{k} \rightarrow \infty$ as $k \rightarrow \infty, m_{k}<\lambda n_{k}, \lambda<1$, $k=1,2,3, \cdots$ (i.e. the series has Hadamard gaps), while every overconvergent series $f(z)=\sum_{n=0}^{\infty} a_{n} z^{n}$ can, in turn, be written in the form $f(z)=g(z)+h(z)$ where the Taylor series $g(z)$ has Hadamard gaps and $h(z)$ is a Taylor series whose radius of convergence is larger than that of $f(z)$. For a summary of results on overconvergence up to 1937 including proofs of Ostrowski's theorems the reader is referred to [1]. It is our purpose in this work to consider some conditions on the distribution of the zeros of the polynomials that stand between the gaps of a "gap series" that are sufficient to imply the overconvergence of the series, and to make an application of such results to a problem in the Fatou-Pólya circle of ideas.

Received by the editors August 5, 1954 and, in revised form, November 18, 1954.

1 This work forms part of the author's Ph.D. dissertation, Harvard University, 1953, written under the direction of Professor J. L. Walsh, to whom the author wishes to express his gratitude for advice and encouragement. The work was also supported, in part, by the Office of Naval Research under contract N5-ori 07634. 
1. The following result due to Walsh [2] is useful in what follows. Let $C_{1}$ and $C_{2}$ denote the disjoint discs $\left|z-A_{1}\right| \leqq r_{1}$ and $\left|z-A_{2}\right| \leqq r_{2}$ respectively. Let $w$ run through all points such that $\left|w-z_{1}\right|=\left|w-z_{2}\right|$ for some $z_{1}$ of $C_{1}$ and $z_{2}$ of $C_{2}$. Then $w$ fills out the closed "exterior" of the hyperbola whose foci are $A_{1}$ and $A_{2}$ and whose "constant difference" is $r_{1}+r_{2}$.

If, in particular, we set $A_{1}=0, A_{2}=1, r_{1}=0, r_{2}=\delta(0<\delta<1)$, the closed "exterior" is the set of $(x, y)$ such that

$$
h(x, y)=\frac{(x-1 / 2)^{2}}{\delta^{2} / 4}-\frac{y^{2}}{\left(1-\delta^{2}\right) / 4}-1 \leqq 0 .
$$

Let $R(\delta)$ denote the open "interior" of the right branch, i.e. the set of $w=x+i y$ with $x>(1+\delta) / 2$ and $h(x, y)>0$. Then the result of Walsh implies the following.

Lemma 1. If $0<\delta<1$ and $C_{2}$ denotes the circle $|1-z| \leqq \delta$, then if $w=x+i y$ and $z \in C_{2}$,

$$
|1-z / w|<1
$$

if, and only if, $w \in R(\delta)$.

Using Lemma 1 we can prove the following:

THEOREM 1. Let $f(z)=\sum_{n=0}^{\infty} a_{n} z^{n}, \lim \sup _{n \rightarrow \infty}\left|a_{n}\right|^{1 / n}=1$. Suppose there exist two strictly monotone increasing sequences of integers $\left\{m_{k}\right\}_{k=1}^{\infty},\left\{n_{k}\right\}_{k=1}^{\infty}$ such that $a_{n}=0$ for $m_{k}<n<n_{k}, k=1,2, \cdots$, and

$$
\begin{gathered}
\limsup _{k \rightarrow \infty}\left|a_{n_{k}}\right|^{1 / n_{k}}=r<1, \\
\limsup _{k \rightarrow \infty} \frac{m_{k}-n_{k-1}}{n_{k-1}}=K>0 .
\end{gathered}
$$

Furthermore, suppose that upon writing $f(z)=\sum_{k=1}^{\infty} z^{n_{k-1}} p_{k}(z)\left(n_{0}=0\right)$ there exists $\delta, 0<\delta<1$, and $d>0$, independent of $k$, such that $N_{k}$ zeros of $p_{k}(z)$ are in $R(\delta), k=1,2, \cdots$,

$$
\liminf _{k \rightarrow \infty} \frac{N_{k}}{m_{k}-n_{k-1}}=\mu,
$$

and the remaining zeros of $p_{k}(z)$ all have moduli greater than or equal to $d$, where $\mu$ and $d$ satisfy

$$
r<(d /(1+d))^{K(1-\mu)} .
$$

Then there exists $\delta_{0}>0$ such that $\left\{S_{m_{k}}(z)\right\}_{k=1}^{\infty}$ converges uniformly in 
$|1-z|<\delta_{0}$ (where $\left.S_{n}(z)=\sum_{k=0}^{n} a_{k} z^{k}\right)$, i.e., $\sum_{n=0}^{\infty} a_{n} z^{n}$ overconverges in a neighborhood of $z=1$.

Before we give the proof let us remark that hypotheses (2) and (3) are necessary conditions for overconvergence (cf. [3]).

PROOF. If the zeros of $p_{k}(z)$ in $R(\delta)$ be denoted by $z_{1}, \cdots, z_{N_{k}}$, we obtain as a consequence of Lemma $1,\left|\left(1-z / z_{1}\right) \cdots\left(1-z / z_{N_{k}}\right)\right|<1$, while the remaining zeros of $p_{k}(z), z_{N_{k+1}}, \cdots, z_{j}, \cdots, z_{m_{k}-n_{k-1}}$ all satisfy $\left|z_{j}\right| \geqq d$. So, if we restrict $z$ to satisfy $|1-z| \leqq \delta_{1}<\delta$, then $\left|1-z / z_{j}\right| \leqq\left(1+d+\delta_{1}\right) / d$ and hence $\left|\left(1-z / z_{N_{k+1}}\right) \cdots\left(1-z / z_{m_{k-n_{k-1}}}\right)\right|$ $\leqq\left(\left(1+d+\delta_{1}\right) / d\right)^{m_{k}-n_{k-1}-N_{k}}$. Furthermore, for $k$ sufficiently large, $\left|a_{n_{k-1}}\right|<(r+\eta)^{n_{k-1}}, \eta>0$, and so

$$
|z|^{n_{k-1}}\left|p_{k}(z)\right|<\left(1+\delta_{1}\right)^{n_{k-1}}(r+\eta)^{n_{k-1}}\left(\frac{1+d+\delta_{1}}{d}\right)^{m_{k}-n_{k-1}-N_{k}}
$$

In view of (3) and (4), (6) implies

$$
|z|^{n_{k-1}}\left|p_{k}(z)\right|<\left(\left(1+\delta_{1}\right)(r+\eta)\left(\frac{1+d+\delta_{1}}{d}\right)^{(K+\eta)(1-\mu+\eta)}\right)^{n_{k-1}},
$$

and if $\delta_{1}$ and $\eta$ be chosen small enough, we have

$$
|z|^{n_{k-1}}\left|p_{k}(z)\right|<b^{n_{k-1}}, \quad 0<b<1,
$$

as a consequence of (5). This establishes the theorem.

We remark that if all the zeros of $p_{k}(z)$ are in some $R(\delta)$, then $\mu=1$ in (4), and (5) is redundant in view of (2). Theorem 1 is illustrated by the classical Porter-Ostrowski example

$$
f(z)=\sum_{k=1}^{\infty} \frac{\{z(1-z)\}^{4 k}}{p_{k}},
$$

where $p_{k}$ is the largest coefficient of $(1+z)^{4^{k}}$. However, the overconvergence of

$$
\sum_{k=1}^{\infty} \frac{\left\{z^{2}\left(1-z^{2}\right)\right\}^{4 k}}{p_{k}}
$$

cannot be established by means of Theorem 1 as (5) does not hold. This example is amenable to the following analogue of Theorem 1.

Theorem 2. Let $f(z)=\sum_{n=0}^{\infty} a_{n} z^{n}$, lim $\sup _{n \rightarrow \infty}\left|a_{n}\right|^{1 / n}=1$. Suppose there exist two strictly monotone increasing sequences of integers $\left\{m_{k}\right\}_{k=1}^{\infty}$ and $\left\{n_{k}\right\}_{k=1}^{\infty}$ such that $a_{n}=0$ for $m_{k}<n<n_{k}, k=1,2, \cdots$, and

$$
\limsup _{k \rightarrow \infty}\left|a_{m_{k}}\right|^{1 / m_{k}}=\rho<1,
$$




$$
0<K=\lim _{k \rightarrow \infty} \frac{m_{k}-n_{k-1}}{n_{k-1}} .
$$

Furthermore, using the notation of Theorem $1, N_{k}$ zeros of $p_{k}(z)$ are in $|1-z| \leqq a, k=1,2, \cdots, 0 \leqq a, a$ independent of $k$,

$$
\epsilon=\lim _{k \rightarrow \infty} \frac{N_{k}}{m_{k}-n_{k-1}}
$$

and the remaining zeros of $p_{k}(z)$ lie in $|z| \leqq M$ where $M$ satisfies

$$
\rho^{K+1} a^{\epsilon K}(1+M)^{(1-\epsilon) K}<1 .
$$

Then there exits $\delta>0$ such that $\left\{S_{m_{k}}(z)\right\}_{k=1}^{\infty}$ converges uniformly in $|1-z|<\delta$.

We add that the requirements that the limit exist in (10) and (11) are made for purposes of simplicity of statement. If $a<1$ the limit in (10) and (11) may be replaced by the inferior limit, while if $a \geqq 1$ it may be replaced by the superior limit. With this modification (9) and (10) are necessary conditions for overconvergence according to [3]. The proof of Theorem 2 is entirely analogous to that of Theorem 1 , save that we use here the factorization of $p_{k}(z)$ in the form $a_{m_{k}}\left(z-z_{1}\right)$ $\cdots\left(z-z_{m_{k-n_{k-1}}}\right)$, and will be omitted.

2. In this section we wish to use Theorems 1 and 2 to give some results in the Fatou-Pólya circle of ideas. It will be recalled that the Fatou Conjecture, first proved by Pólya [4], states that every Taylor series of finite nonzero radius of convergence can be made to have its circle of convergence as a natural boundary by changing the signs of a denumerable number of properly chosen coefficients. Steinhaus [5] has proved that given the Taylor series $\sum_{n=1}^{\infty} c_{n} z^{n}$ with radius of convergence 1 , then among the series $\sum_{n=1}^{\infty} c_{n} e^{2 \pi i \phi_{n}} z^{n}$, where the $\phi_{n}$ are independent of one another, those that are analytically continuable in some direction beyond the unit circle form a set of measure zero. It is our purpose in this section to exhibit representatives of this set of measure zero for series of a certain type.

A sequence of real numbers $\left\{\phi_{n}\right\}_{n=0}^{\infty}$ such that $0 \leqq \phi_{n}<2 \pi$, $n=0,1,2, \cdots$, will be called an argument sequence.

THEOREM 3. Let $f(z)=\sum_{n=0}^{\infty} a_{n} z^{n}$, lim sup $\left|a_{n}\right|^{1 / n}=1$. Suppose there exist two strictly monotone increasing sequences of integers $\left\{m_{k}\right\}_{k=1}^{\infty}$, $\left\{n_{k}\right\}_{k=1}^{\infty}$ such that $a_{n}=0$ for $m_{k}<n<n_{k}, k=1,2, \cdots$, and

$$
\limsup _{k \rightarrow \infty} \frac{m_{k}-n_{k-1}}{n_{k-1}}=K>0 \text {. }
$$


Furthermore, using the notation of Theorem 1, suppose there exists $\eta>0$ such that for some fixed positive integer $m \geqq 3, p_{k}(z), k=1,2, \cdots$, has all its zeros in $|z| \geqq(\sec \pi / m) / 2+\eta=d(m)+\eta$, and

$$
\limsup _{k \rightarrow \infty}\left|a_{n_{k}}\right| 1 / n_{k}=r<\left(\frac{d(m)}{1+d(m)}\right)^{K((m-1) / m)}
$$

Then, if $\phi$ is a real number satisfying $0 \leqq \phi<2 \pi$ there exists an argument sequence $\left\{\phi_{n}\right\}_{n=0}^{\infty}$, depending only on $\phi$, and a $\delta>0$ such that the sequence $\left\{S_{m_{k}}^{*}(z)\right\}_{k=1}^{\infty}$, where $S_{m_{k}}^{*}(z)=\sum_{n=0}^{m_{k}} a_{n} e^{i \phi_{n} z^{n}}$, converges uniformly in $\left|e^{i \phi}-z\right|<\delta$.

Proof. Let $C$ be the circle $|z|=1 / 2$ and let $\Gamma_{m}$ be the regular polygon of $m$ sides circumscribing $C$ and such that the point $z=1 / 2$ is a midpoint of one of the sides. Consider the $m$ point sets $A_{0}, \cdots, A_{m-1}$ where $z \in A_{j}\left(e^{2 \pi j i / m} / 2, q, a\right)$ if, and only if, $\left|\arg \left(z-e^{2 \pi j i / m} / 2\right)\right| \leqq a$ $<\pi / 2$ and $\operatorname{Re}\left(z e^{-2 \pi j i / m}\right) \geqq 1 / 2+q, q>0$, with $q$ and $a$ chosen as follows. The greatest distance of a point of $\Gamma_{m}$ from the origin is $2^{-1} \sec (\pi / m)>1 / 2$. We choose $q$ and $\pi / 2-a$ so small that any point of $|z| \geqq 2^{-1} \sec (\pi / m)+\eta$ lies in $\sum_{j=0}^{m-1} A_{j}=A$.

Now $p_{k}(z)$ has $m_{k}-n_{k-1}$ zeros in $A, k=1,2, \cdots$. Therefore, for each $k$ there exists at least one integer $t(k), 0 \leqq t(k) \leqq m-1$, such that if $N_{k}$ denotes the number of zeros in $A_{t(k)}$ of $p_{k}(z)$

$$
\frac{N_{k}}{m_{k}-n_{k-1}} \geqq \frac{1}{m}
$$

We so choose a specific $t(k)$ for each $k$. Put $\phi_{n}=n(2 \pi t(k) / m-\phi)$ for $n_{k-1} \leqq n \leqq m_{k}, k=1,2, \cdots$, put $b_{k}(z)=z^{n_{k-1}} p_{k}(z)$ then

$$
b_{k}^{*}(z)=\sum_{n=n_{k-1}}^{m_{k}} a_{n} e^{i \phi_{n}} z^{n}=b_{k}\left(e^{(2 \pi t(k) / m-\phi) i} z\right) .
$$

The rigid rotation of the plane $z \rightarrow e^{(\phi-2 \pi+t(k) / m) i} z$ takes $A_{t(k)}$, $k=1,2, \cdots$, into the set $S\left(e^{i \phi} / 2, q, a\right)$ where $z \in S$ if, and only if, $\left|\arg \left(z-e^{i \phi} / 2\right)\right| \leqq a<\pi / 2$ and $\operatorname{Re}\left(z e^{-i \phi}\right) \geqq 1 / 2+q$. Thus $b_{\boldsymbol{k}}^{*}(z)$ has $N_{k}$ zeros in $S$, for each $k$. Hence $p_{k}^{*}(z)=b^{*}(z) / z^{n_{k-1}}$ also has $N_{k}$ zeros in $S$ for each $k$, and furthermore

$$
\liminf _{k \rightarrow \infty} \frac{N_{k}}{\left(m_{k}-n_{k-1}\right)}=\mu \geqq \frac{1}{m},
$$

while the remaining zeros of $p_{\boldsymbol{k}}^{*}(z)$ all have moduli greater than $d(m)$. (14) implies 


$$
r<\left(\frac{d(m)}{1+d(m)}\right)^{K(1-\mu)}
$$

in view of (15). Finally we note that $S$ is contained in a region $R(\delta)$ (with reference to the point $z=e^{i \phi}$ rather than $z=1$ as in Theorem 1 ; this modification is trivial) for some sufficiently small $\delta>0$, and so the result follows as a consequence of Theorem 1 , where the point $z=1$ is replaced by $z=e^{i \phi}$.

To illustrate Theorem 3 we consider the Taylor series

$$
\sum_{n=1}^{\infty} \frac{\left\{z\left(w_{n}-z\right)\right\}^{4^{n}}}{p_{n}},
$$

where $p_{n}$ is the largest coefficient of $(1+z)^{4^{n}}$, and $\left\{w_{n}\right\}_{n=1}^{\infty}$ consists of all roots of unity enumerated as follows. Let $w_{q, 1}, \cdots, w_{q, q}$ be the $q$ th roots of unity arranged in order of ascending argument. We put $w_{1}=w_{1,1}, w_{2}=w_{2,1}, w_{3}=w_{2,2}, w_{4}=w_{3,1}, w_{5}=w_{3,2}$, and so forth. This series has the unit circle as circle of convergence and also as a natural boundary. A successful argument sequence, whose existence is affirmed by Theorem 3 , is seen to be $\phi_{n}=n\left(\arg w_{k}-\phi\right), n_{k-1} \leqq n \leqq m_{k}$, $k=1,2, \cdots, n=1,2, \cdots$.

It is quite evident that a result similar to that of Theorem 3 can be obtained by using Theorem 2 in place of Theorem 1 in order to establish overconvergence.

\section{BiBLIOGRAPHY}

1. G. Bourion, L. Ultraconvergence dans les sêries de Taylor, Actualités Scientifiques et Industrielles, no. 472, Paris, Hermann, 1937.

2. J. L. Walsh, $A$ certain two dimensional locus, Amer. Math. Monthly vol. 29 (1922) pp. 112-114.

3. - Note on the coefficients of overconvergent power series, Bull. Amer. Math. Soc. vol. 48 (1942) pp. 163-166.

4. G. P6lya and A. Hurwitz, Zwei Beweise eines von Herrn Fatou vermuteten Satzes, Acta Math. vol. 40 (1916) pp. 179-183.

5. H. Steinhaus, Über die Wahrscheinlichkeit dafür, dass der Konvergenzkreis einer Potenzreihe ihre natiurliche Grenze ist, Math. Zeit. vol. 31 (1929) pp. 408-416.

HARVARD UNIVERSITY 